\title{
Transient Structures of PdO During CO Oxidation over $\operatorname{Pd}(100)$
}

Mikhail Shipilin, ${ }^{\dagger}$ Johan Gustafson, ${ }^{\dagger}$ Chu Zhang, ${ }^{\dagger}$ Lindsay R. Merte $^{\dagger}$ Andreas Stierle,, Uta Hejral, Per-Anders Carlsson, ${ }^{\S}$ and Edvin Lundgren ${ }^{* \dagger} \dagger$

Division of Synchrotron Radiation Research, Lund University, SE-22100 Lund, Sweden, University of Hamburg, D-20355 Hamburg, Germany, Deutsches Elektronen-Synchrotron (DESY), D-22603 Hamburg, Germany, and Competence Centre for Catalysis, Chalmers University of Technology, SE-41296 Göteborg, Sweden

E-mail: edvin.lundgren@sljus.lu.se

\footnotetext{
${ }^{*}$ To whom correspondence should be addressed

${ }^{\dagger}$ Lund University

${ }^{\ddagger}$ Hamburg University

IDESY

${ }^{\S}$ CCC Chalmers
} 


\begin{abstract}
In situ high-energy surface X-ray diffraction was employed to determine the surface structure dynamics of a $\operatorname{Pd}(100)$ single crystal surface acting as a model catalyst to promote $\mathrm{CO}$ oxidation. The measurements were performed under semi-realistic conditions, i.e. 100 mbar total gas pressure and $600 \mathrm{~K}$ sample temperature. The surface structure was studied in detail both in a steady gas flow and in a gradually changing gas composition with a time resolution of $0.5 \mathrm{sec}$. The experimental technique allows for rapid reciprocal space mapping providing the complete information on structural changes of a surface with unprecedented time resolution in harsh conditions. Our results show that the $(\sqrt{5} \times \sqrt{5}) \mathrm{R} 27^{\circ}-\mathrm{PdO}(101)$ surface oxide forms in a close to stoichiometric $\mathrm{O}_{2}$ and $\mathrm{CO}$ gas mixture as the mass-spectrometry indicates a transition to a highly active state with the reaction rate limited by the $\mathrm{CO}$ mass transfer to the $\operatorname{Pd}(100)$ surface. Using a low excess of $\mathrm{O}_{2}$ in the gas stoichiometry, islands of bulk oxide grow epitaxially in the same (101) crystallographic orientation of the bulk PdO unit cell according to a Stranski-Krastanov type of growth. The morphology of the islands is analyzed quantitatively. Upon further increase of the $\mathrm{O}_{2}$ partial pressure a polycrystalline $\mathrm{Pd}$ oxide forms on the surface.
\end{abstract}

\title{
Keywords
}

HESXRD, SXRD, Pd oxidation, heterogeneous catalysis, X-ray diffraction

\section{Introduction}

For more than a century heterogeneous catalysis has been extensively exploited by the industry, and as a consequence it has been intensively studied. ${ }^{1}$ One of the most prominent examples is the $\mathrm{CO}$ oxidation reaction, $\mathrm{CO}+{ }^{1} /{ }_{2} \mathrm{O}_{2} \rightarrow \mathrm{CO}_{2}$. This process transforms highly toxic carbon monoxide, formed e.g. as a by-product during incomplete combustion of the fuel in internal combustion engines, to less harmful carbon dioxide gas. However, the reaction is 
very slow under the operational conditions in gas-phase, and requires thus the presence of a solid catalysts to proceed at a sufficiently high rate. Because of its importance and relatively simple mechanism this reaction has become the subject of numerous studies aiming to resolve the atomic-scale processes that occur on the surface of catalysts. ${ }^{2}$

Supported nanoparticles of late transition metals represent a well-known and efficient type of oxidation catalyst, and are currently widely used in catalytic converters. ${ }^{3,4}$ Hence, a deep understanding of the fundamental processes proceeding in such systems is important for improvement of existing catalyst-based solutions and development of new potential approaches. For this purpose, studies of atomic-scale surface structure and determination of the active phase of catalysts under working conditions is essential. However, the complexity of such systems and the inability of many experimental techniques to work under realistic pressures - the challenges known as material and pressure gaps - significantly narrow the selection of available methods for structural determination and necessitate the use of model systems. One of the commonly used approaches is to study single crystals with different surface crystallographic orientations in order to reproduce the behavior of corresponding nanoparticle facets. ${ }^{5}$ In the case of catalytic oxidation, in particular $\mathrm{CO}$ oxidation, different oxide phases forming on the surface are in many cases considered to be responsible for the catalytic activity. ${ }^{6}$ This fact emphasizes the importance of detailed surface structure investigations.

Palladium-based catalysts are known to show high performance for oxidation of $\mathrm{CO}$ and hydrocarbons. Over the last years the surface structure of Pd single crystals with different crystallographic orientations $^{7-11}$ and in particular $\operatorname{Pd}(100)^{12-15}$ has been extensively studied theoretically and experimentally under $\mathrm{O}_{2}$ exposure at different pressures both ex situ and in situ.

Previously both conventional in situ surface X-ray diffraction (SXRD) ${ }^{16}$ and high-pressure X-ray photoelectron spectroscopy (HPXPS $)^{17}$ have been used to study CO oxidation over $\operatorname{Pd}(100)$ under semi-realistic conditions. Despite differences in reactor chamber geometries 
and obtainable pressures, both studies revealed CO diffusion limitations as the $\operatorname{Pd}(100)$ model catalyst becomes active. This behavior significantly changes the gas composition close to the sample surface, ${ }^{17,18}$ and if either the oxygen partial pressure or the total pressure of the gas mixture in the reactor is high enough, the surface will be oxidized.

Recently we demonstrated the ability of high-energy SXRD to follow surface structural changes of model catalysts in situ under realistic conditions with a time resolution sufficient for observing surface dynamics during catalytic processes. ${ }^{19}$ We also reported on the structure of the surface oxide forming on $\operatorname{Pd}(100)$ when it becomes catalytically active in a close to stoichiometric mixture of reactants for the reaction of $\mathrm{CO}$ oxidation. ${ }^{20}$ Epitaxial growth of $\mathrm{PdO}(101)$ on $\mathrm{Pd}(100)$ has been reported theoretically ${ }^{15}$ as well as experimentally in pure oxygen $^{21}$ and in a mixture of $\mathrm{CO}$ and oxygen. ${ }^{22} \mathrm{~A}$ disordered polycrystalline $\mathrm{PdO}$ film has also been reported using pure oxygen. ${ }^{22,23}$ However the transition between the different oxide phases during $\mathrm{CO}$ oxidation has not previously been reported.

In the present report, we have used high-energy SXRD to study the surface structure of $\operatorname{Pd}(100)$ in situ during $\mathrm{CO}$ oxidation. Our study confirms that three different types of $\mathrm{Pd}$ oxides can be observed in the highly active state of the model catalyst, depending on the $\mathrm{O}_{2}$ partial pressure: a $(\sqrt{5} \times \sqrt{5}) \mathrm{R} 27^{\circ}$ surface oxide, epitaxial $\mathrm{PdO}$ islands co-existing with the surface oxide and a polycrystalline $\mathrm{PdO}$ film. These phases are consistent with previous observations, both under purely oxidizing conditions ${ }^{21}$ and under semi-realistic reaction conditions. ${ }^{22}$ In this work we compile the qualitative and quantitative information about the different oxide phases and highlight the structural transitions of the surface in concert with simultaneous measurements of the catalytic activity. The structural information extracted from a large part of the reciprocal space recorded during the experiment allows us to monitor and exclude the potential presence of other ordered $\mathrm{PdO}$ structures except those mentioned above. 


\section{Experimental}

To perform the diffraction experiments we employed synchrotron radiation with an energy of $85 \mathrm{keV}$ at beamline P07 at PETRA III, Deutsches Elektronen-Synchrotron (DESY) in Hamburg, Germany. In order to minimize the penetration depth of the incoming radiation and thus to maximize the ratio between the surface and the bulk signals the angle of incidence was set to $0.04^{\circ}$, which is close to the critical angle of total external reflection for palladium when using $85 \mathrm{keV}$ X-rays.

The sample was placed in a specially designed chamber ${ }^{24}$ mounted on a diffractometer, which allows for a precise surface alignment to the incident beam. ${ }^{25}$ The setup combines an ultra-high vacuum (UHV) chamber for sample preparation with a gas flow reactor where the sample can be exposed to a gas mixture at a total pressure of up to 1 bar. The flows of the gases were separately controlled by mass-flow controllers. The partial pressures of the reactants and the reaction products were followed with a residual gas analyzer.

Before the measurements the sample was cleaned by cycles of $\mathrm{Ar}^{+}$sputtering at $1.5 \mathrm{keV}$ for 20 minutes in a $5 \times 10^{-5}$ mbar Ar gas pressure and annealed up to $1000 \mathrm{~K}$ until the diffraction pattern indicated a clean $\operatorname{Pd}(100)$ surface. After that the reactor volume with the sample inside was isolated from the UHV chamber and a mixture of $\mathrm{O}_{2}, \mathrm{CO}$ and $\mathrm{Ar}$ was introduced into the reactor in a controlled way allowing for changes in the partial pressure of the reactants. The total pressure of the gas mixture was set to $100 \mathrm{mbar}$ and the temperature of the sample surface was kept at $600 \mathrm{~K}$ throughout the entire experiment.

Possibly present metal carbonyls were removed from the $\mathrm{CO}$ gas by means of an active carbonyl trap before mixing the $\mathrm{CO}$ with other gases.

During the experiment the sample was continuously exposed to a photon flux in the order of $5 \times 10^{10}$ photons/sec with an incident beam size of $2 \times 40 \mu \mathrm{m}^{2}$. The diffraction pattern did not show any changes with the time during exposure indicating that there were no effects connected to beam damage.

A tetragonal basis set of vectors $\mathbf{a}_{1}, \mathbf{a}_{2}$ lying in the surface plane and $\mathbf{a}_{3}$ perpendicular 
to them was used to describe the crystal structure. In terms of the bulk lattice constant $a_{0}(P d)=3.89 \AA$, the lengths of these vectors can be expressed as $\left|\mathbf{a}_{1}\right|=\left|\mathbf{a}_{2}\right|=a_{0} / \sqrt{2}$ and $\left|\mathbf{a}_{3}\right|=a_{0}$.

To record the diffraction patterns a $410 \times 410 \mathrm{~mm}^{2}$ Perkin-Elmer flat panel X-ray detector was used. It is adapted for energies above $20 \mathrm{keV}$ and has a resolution of 4-megapixel with $200 \times 200 \mu \mathrm{m}^{2}$ physical size of an individual pixel. The high intensity of the bulk diffraction maxima may cause oversaturation and damage to the detector. In order to avoid that, the corresponding parts of the detector were covered with Densimet ${ }^{\circledR}$ pieces, which absorb the electromagnetic radiation. To remove the radiation scattered from the Be walls of the reactor from resulting diffraction patterns, a specially designed tungsten mask was placed at a short distance from the sample blocking the corresponding part of the outgoing radiation. Both the Densimet ${ }^{\circledR}$ protection and the mask cause shaded areas in the diffraction patterns, which were excluded from the analysis.

In the current experiment each recorded diffraction pattern corresponds to a slice in the reciprocal space at a fixed sample azimuth and spans over the area from -5 to $5 \AA^{-1}$ and from 0 to $5 \AA^{-1}$ in in-plane and out-of-plane directions, respectively. In the case of $\operatorname{Pd}(100)$ this part contains diffraction peaks with $h$ and $k$ values up to 2 and $l$ values up to 3 reciprocal lattice units (RLU). In order to collect three dimensional information about the system under study the crystal is rotated in the photon beam under constant incident angle and the detector records the diffraction patterns corresponding to different azimuthal angles. In the case of $\operatorname{Pd}(100)$, a $45^{\circ}$ rotation is sufficient because it reveals all characteristic features of the system with the p4mm symmetry. In our experiment we recorded a $90^{\circ}$ rotation range. The angular step was chosen to be $0.1^{\circ}$ resulting in 900 diffraction images in one data set. The details of data recording and treatment can be found in ref. ${ }^{20}$ 


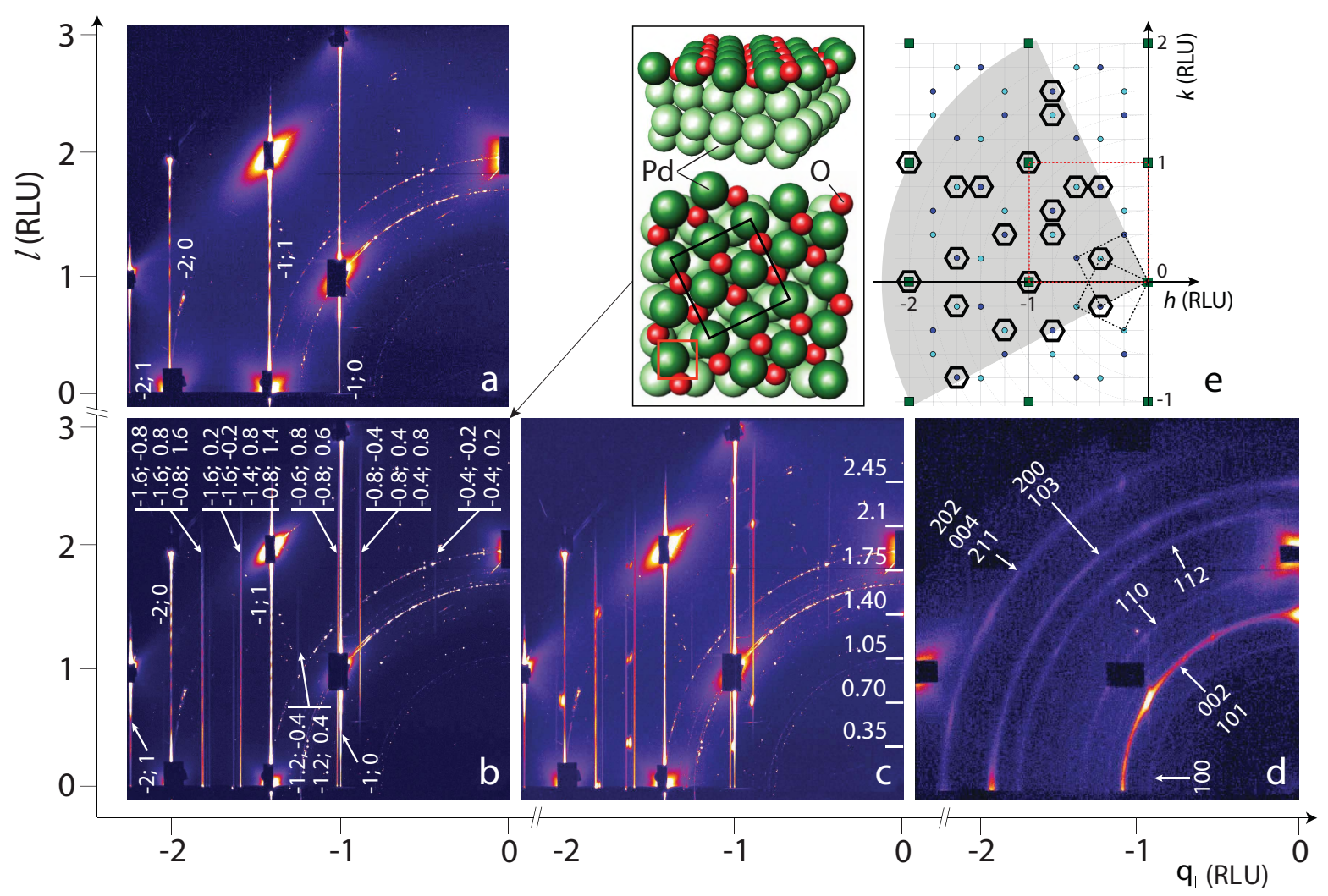

Figure 1: (Color online) Images obtained by taking the highest value of the intensity recorded for each pixel position after comparison of all images of the four HESXRD data sets obtained for $90^{\circ}$ rotation under the incoming photon beam while the $\operatorname{Pd}(100)$ single crystal acts as a model catalyst during CO oxidation at 100 mbar total gas pressure at $600 \mathrm{~K}$ (a) under CO rich conditions, (b) in close to stoichiometric mixture of $\mathrm{CO}$ and $\mathrm{O}_{2}$, (c) under conditions with a relatively low excess of $\mathrm{O}_{2}$, (d) under conditions with a relatively high excess of $\mathrm{O}_{2}$. Note that the in-plane positions of the diffraction rods are absent in the two-dimensional images. They are instead shown in the in-plane reciprocal map (e) along with the ball model of the surface $\mathrm{PdO}(101)$ to demonstrate the correspondence of the in-plane periodicity of the diffraction patterns to the relative size and orientation of $\operatorname{Pd}(100)$ (indicated by a red square) and the $(\sqrt{5} \times \sqrt{5}) \mathrm{R} 27^{\circ}-\mathrm{PdO}(101)$ surface oxide (indicated by a black square) unit cells. Two dashed-line black squares show the hk-basis of the diffraction patterns caused by two mirror domains of the surface structure. The grey shape shows the recorded part of the reciprocal space. Black hexagons indicate the diffraction rods that we observe in our experiment. Their in-plane positions are shown in panels (a-b). Crystallographic planes producing visible diffraction rings are indicated in the panel (d). 


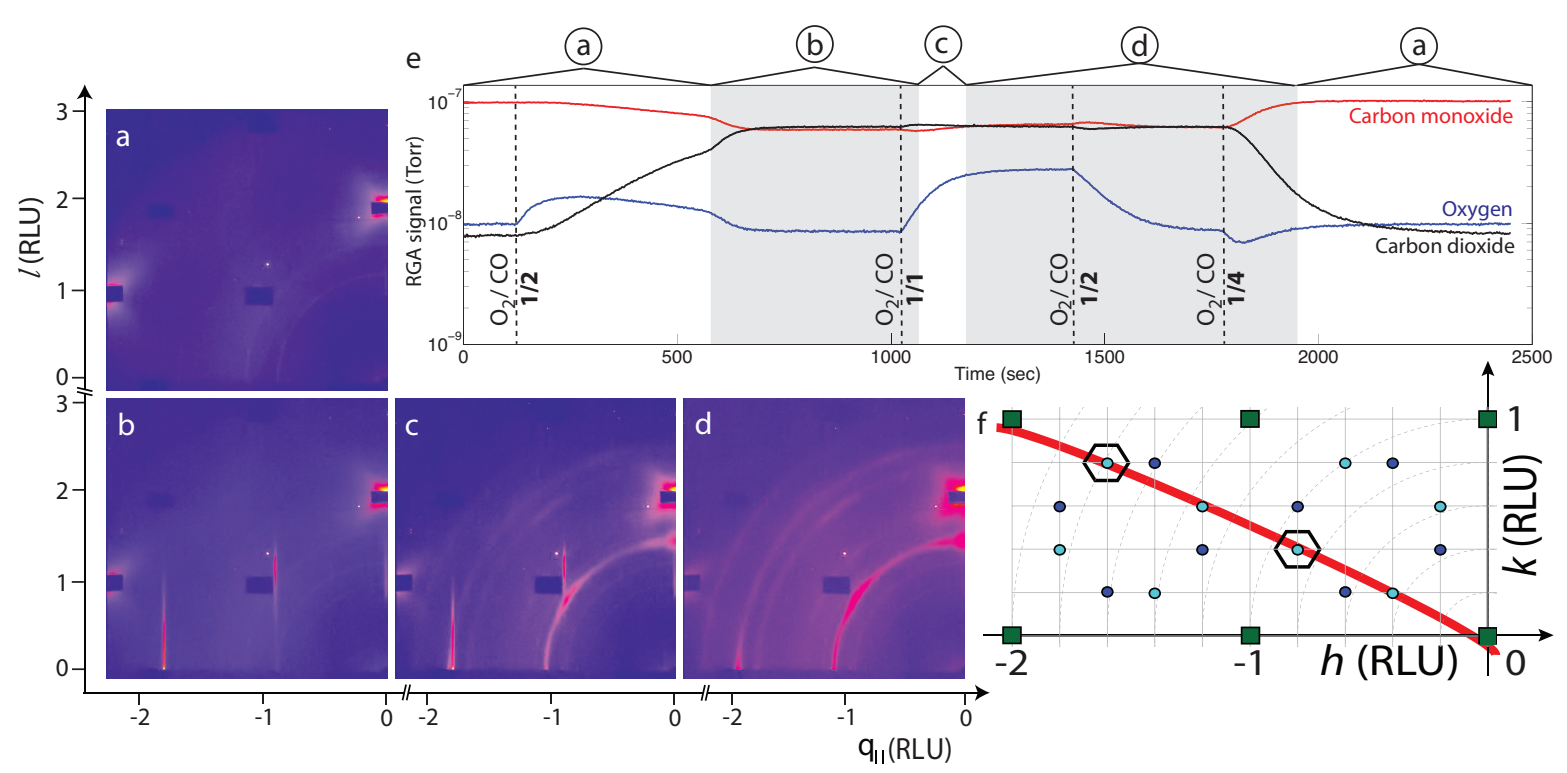

Figure 2: (Color online) (a-d) diffraction patterns observed at the fixed azimuthal sample position where the part of the reciprocal space corresponding to the surface of the Ewald sphere (indicated by the red curved line in the reciprocal map (f)) can be observed. (e) the mass spectrometer signal indicating the composition of the gas mixture in the reactor. Dashed vertical lines in the mass spectrometer data indicate the moments when the gas flow was changed; encircled letters indicate the intervals where the respective diffraction patterns (a-d) were continuously observed.

\section{Results}

The diffraction patterns were collected under a steady flow using four different gas mixtures: 1) about $1 \mathrm{ml}_{n} / \mathrm{min} \mathrm{O}_{2}\left(1 \mathrm{ml}_{n}\right.$ is the amount of gas corresponding to $1 \mathrm{ml}$ at standard pressure and temperature), $4 \mathrm{ml}_{n} / \min \mathrm{CO}$ and $25 \mathrm{ml}_{n} / \mathrm{min}$ Ar, i.e. with an excess of $\mathrm{CO}$; 2) about $2 \mathrm{ml}_{n} / \min \mathrm{O}_{2}, 4 \mathrm{ml}_{n} / \min \mathrm{CO}$ and $25 \mathrm{ml}_{n} / \min \mathrm{Ar}$, i.e. close to the stoichiometric ratio between $\mathrm{CO}$ and $\mathrm{O}_{2}$ for complete oxidation of $\mathrm{CO}$ to $\mathrm{CO}_{2} ; 3$ ) in a flow of about 2.3 $\mathrm{ml}_{n} / \min \mathrm{O}_{2}, 4 \mathrm{ml}_{n} / \min \mathrm{CO}$ and $25 \mathrm{ml}_{n} / \min \mathrm{Ar}$, i.e. with a small excess of $\left.\mathrm{O}_{2} ; 4\right)$ in a flow of about $4 \mathrm{ml}_{n} / \min \mathrm{O}_{2}, 4 \mathrm{ml}_{n} / \min \mathrm{CO}$ and $25 \mathrm{ml}_{n} / \mathrm{min} \mathrm{Ar}$, i.e. with a relatively large excess of $\mathrm{O}_{2}$.

Fig. 1(a-d) show images obtained by taking the highest value of the intensity recorded for each pixel position after comparison of all images of the entire data set for the sets recorded for the $\mathrm{Pd}(100)$ crystal surface under the four gas conditions specified above (1-4 respectively). Under the $\mathrm{CO}$ rich condition the diffraction pattern (see Fig. 1(a)) reveals 
only $\operatorname{Pd}(100)$ crystal truncation rods corresponding to the metallic state of the surface while the mass spectrometer does not show any significant $\mathrm{CO}_{2}$ production. We assign this to $\mathrm{CO}$ self-poisoning of the reaction.

Under stoichiometric conditions for complete $\mathrm{CO}$ oxidation a more intricate diffraction pattern is recorded (see Fig. 1(b)). The fact that some diffraction rods are observed at positions that do not correspond to the $\operatorname{Pd}(100)$ crystal truncation rods reveals the presence of a surface superstructure with an in-plane periodicity different from the substrate. We have previously described this superstructure in detail and concluded that it is a $(\sqrt{5} \times \sqrt{5}) \mathrm{R} 27^{\circ}$ $\mathrm{PdO}(101)$ surface oxide, which is represented by a $\mathrm{PdO}(101)$ monolayer. ${ }^{19,20}$ A ball model of this structure is shown in Fig. 1(e) together with a corresponding in-plane reciprocal map. The diffraction rods that are observed in the experiment are marked on this map by black hexagons while others are not observed because of the too low structure factor value. The mass spectrometer shows a high catalytic activity in terms of $\mathrm{CO}_{2}$ production under these conditions.

In Fig. 1(c) the diffraction data recorded for the case of low excess of $\mathrm{O}_{2}$ is shown. The mass spectrometry shows a high catalytic activity of the catalyst, however, the partial pressure of the produced $\mathrm{CO}_{2}$ does not change in comparison with the previous gas flow settings. This behavior is assigned to the fact that the reaction is mass-transfer limited with respect to $\mathrm{CO}$. It is clear that the diffraction pattern has changed as compared to the pattern shown in Fig. 1(b). Although the diffraction rods from the $(\sqrt{5} \times \sqrt{5}) \mathrm{R} 27^{\circ}-\mathrm{PdO}(101)$ surface structure are still visible, relatively sharp spots at or in the vicinity of these rods are clearly observed. This indicates the growth of an ordered bulk structure coexisting with the surface oxide and exhibiting the same in-plane periodicity, which is characteristic for epitaxial growth.

Finally, in Fig. 1(d) the image recorded under $\mathrm{O}_{2}$ rich conditions is shown. The $\mathrm{CO}$ conversion to $\mathrm{CO}_{2}$ is still limited by the diffusion of $\mathrm{CO}$ and the mass spectrometer signal for $\mathrm{CO}_{2}$ remains at the same level. The diffraction pattern is significantly different from 
those observed in Fig. 1(a-c). The appearance of diffraction rings indicates the formation of a disordered powder-like film on the surface.

In addition to the above discussed measurements performed in a steady gas flow regime at different relative concentrations of the reactants, we also followed the surface transformation dynamics under changing gas flow conditions. For that purpose the azimuthal position of the sample was fixed at the angle where diffraction features from the oxide structures could be seen. The observable part of reciprocal space in this case approximately corresponds to the part of the Ewald sphere shown in Fig. 2(f). Diffraction patterns were recorded with the speed of one frame per 0.5 second while changing the gas composition in the reactor. The flow of $\mathrm{CO}$ was fixed at $4 \mathrm{ml}_{n} / \mathrm{min}$ and the flow of $\mathrm{O}_{2}$ gradually changed from deficiency to excess of oxygen relatively to the reaction stoichiometry, keeping the total pressure constant.

In Fig. 2(a-d) we show the diffraction patterns recorded for the surface structure of the $\mathrm{Pd}(100)$ single crystal while stepwise changing the $\mathrm{O}_{2}$ :CO partial pressure ratio in the flow into the reactor from 1:4 to $1: 1$ and back. In the gas mixture with 1:4 ratio the detector reveals the diffraction pattern characteristic for the metallic $\operatorname{Pd}(100)$ surface. The crystal truncation rods are not observed at this azimuthal sample position because they do not intersect the surface of the Ewald sphere (see Fig. 2(f)) but the $\operatorname{Pd}(100)$ Bragg reflections are visible because of their significant width in hk-plane. The mass spectrometer signal in Fig. 2(e) shows, at the same time, that no significant amounts of $\mathrm{CO}_{2}$ are produced. The activation of the model catalyst occurs when the reactant ratio is changed to 1:2, i.e close to stoichiometric, by increasing the $\mathrm{O}_{2}$ flow. The mass spectrometer signal shows the increase in $\mathrm{CO}_{2}$ production, which reaches a plateau when the reaction reaches the masstransfer limited regime. At the same time two superstructure diffraction rods with (-0.8; $0.4)$ and $(-1.6 ; 0.8)$ in-plane reciprocal coordinates appear in the diffraction pattern. This fact indicates that under these conditions the surface is covered with the $(\sqrt{5} \times \sqrt{5}) \mathrm{R} 27^{\circ}$ $\mathrm{PdO}(101)$ surface oxide. After a further increase of the $\mathrm{O}_{2}$ partial pressure the reactant ratio reaches a 1:1 value. The catalytic activity of the sample remains on the same level because 
of the mass-transfer limited reaction rate. The diffraction data at the same time show the coexistence of the surface oxide structure with polycrystalline PdO indicated by the presence of the diffraction rings. This is a transition state, which rapidly switches to a state where the surface is completely covered with a polycrystalline oxide film. We do not observe the epitaxial growth of $\mathrm{PdO}$ on top of the surface oxide in this case because the fixed azimuthal position of the sample that was chosen here does not allow us to record bulk PdO reflections, as observed in the rotational scan (Fig. 1(c)) and which appear at a slightly different angle. Upon gradual decrease in $\mathrm{O}_{2}$ partial pressure the surface remains covered by a polycristalline $\mathrm{PdO}$ film until the gas flow becomes $\mathrm{CO}$ rich and the surface directly turns to the highly ordered metallic state when reduced by $\mathrm{CO}$. The last observation is interesting because it is not self-evident that a rough surface covered with polycrystalline oxide phase easily recovers to the well ordered state upon the oxide reduction.

It should be mentioned that in our HESXRD data we do not observe any signatures of any disordered phases on the surface within the signal to noise ratio. Besides it, the previous studies of $\mathrm{Pd}(100)$ exposed to oxygen under different conditions using various experimental techniques, to the best of our knowledge, have not revealed any stable disordered formations. These facts with a high certainty allow us not to consider the presence of a significant amount of disordered phases, which would influence the catalytic activity of the surface. In addition, the observed correlation between the structural information obtained in the experiments under different conditions and catalytic activity of the sample strongly suggests that it is the ordered phases discussed in this work that are responsible for catalytic CO oxidation.

\section{Discussion}

Several states of the surface under conditions relevant to the process of CO oxidation have been reported in the literature. Under $\mathrm{CO}$ rich reaction conditions the surface is covered with adsorbed $\mathrm{CO}$ molecules, which hinder the oxygen adsorption and dissociation and thus the 
reaction inhibits itself. ${ }^{17}$ In our HESXRD experiment we refer to that state as the metallic surface and the diffraction data show Bragg peaks and crystal truncation rods characteristic for $\operatorname{Pd}(100)$. With the increase of the oxygen partial pressure the surface has been reported to go through surface oxide covered, epitaxial bulk oxide covered and disordered powder-like oxide covered states. ${ }^{15,21,22}$

At close to stoichiometric ratio of reactants the $\operatorname{Pd}(100)$ surface is covered with the $(\sqrt{5} \times \sqrt{5}) \mathrm{R} 27^{\circ}-\mathrm{PdO}(101)$ surface oxide that previously has been reported and described in detail under different reaction conditions including our recent HESXRD studies carried out under semi-realistic conditions for the process of CO oxidation. ${ }^{19,20}$

The bulk oxide growth, although previously reported in the literature, is less studied and to our knowledge no quantitative determinations have been performed for that structure formed under realistic reaction conditions. In Fig. 3(a-d) the diffraction pattern recorded in a steady gas flow with a small excess of $\mathrm{O}_{2}$ is shown along with the calculated positions for the bulk $\mathrm{PdO}$ reflections including three different crystallographic orientations of growth namely [101] PdO $\|[100] \mathrm{Pd},[100] \mathrm{PdO}\|[100] \mathrm{Pd}$ and [001]PdO $\|[100] \mathrm{Pd}$. As mentioned above, the diffraction pattern is similar to that of the $(\sqrt{5} \times \sqrt{5}) \mathrm{R} 27^{\circ}-\mathrm{PdO}(101)$ surface oxide but with additional Bragg reflections clearly suggesting the presence of the bulk PdO structure, due to the excess of oxygen. Moreover, the fact that the spots are relatively sharp and have very similar in-plane periodicity as the surface oxide diffraction rods indicates that the $\mathrm{PdO}$ grows epitaxialy on the $\operatorname{Pd}(100)$ surface. Further, the observation that the $(\sqrt{5} \times \sqrt{5}) \mathrm{R} 27^{\circ}$ $\mathrm{PdO}(101)$ rods are still present indicates that the epitaxial $\mathrm{PdO}$ grows as islands according to the Stranski-Krastanov mechanism of growth (see Fig. 3(f)).

In ref., ${ }^{15}$ the authors investigate theoretically the transition from the surface oxide to the bulk oxide on $\mathrm{Pd}(100)$ exposed to pure oxygen by employing first-principles calculations based on density functional theory. According to their theoretical model, Stranski-Krastanov type of growth is energetically favored compared to layer-by-layer Frank-van der Merwe growth or three-dimensional islands Vollmer-Weber growth. Our diffraction data show that 


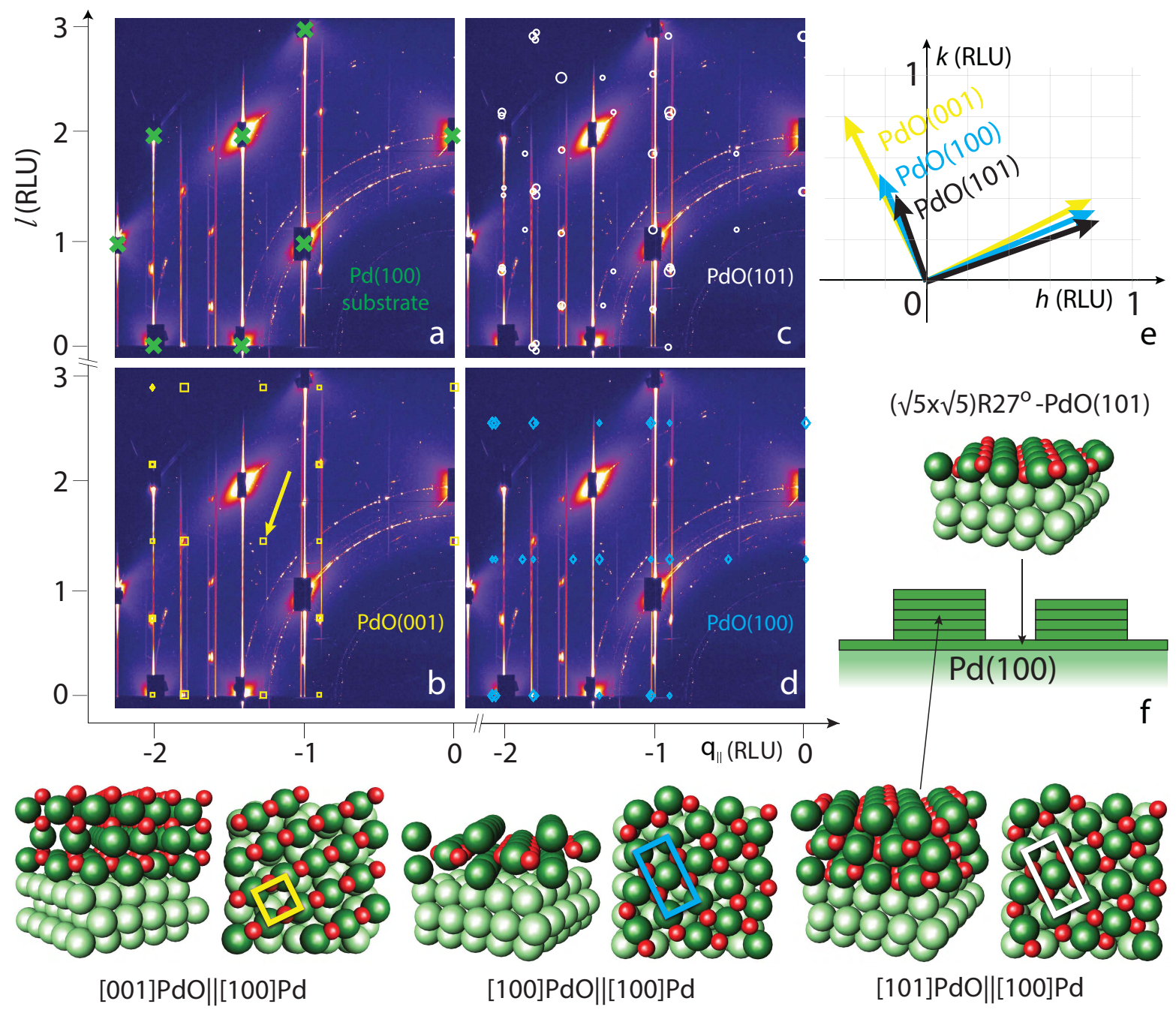

Figure 3: (Color online) Image obtained by taking the highest value of the intensity recorded for each pixel position after comparison of all images of the HESXRD data set obtained for $90^{\circ}$ rotation of the $\mathrm{Pd}(100)$ single crystal in a flow of about $2.3 \mathrm{ml}_{n} / \mathrm{min}_{2}, 4 \mathrm{ml} n / \operatorname{min~CO}$ and $25 \mathrm{ml}_{n} / \mathrm{min}$ Ar, i.e. with a low excess of $\mathrm{O}_{2}, 100 \mathrm{mbar}$ total gas pressure at $600 \mathrm{~K}$ (a-d). Expected positions of the diffraction maxima for three different crystallographic directions of epitaxial PdO growth (the corresponding ball models with the indicated unit cells are shown at the bottom) are marked in the image (different size of tokens indicate the difference in structure factor for different reflections with the smallest size corresponding to the weakest reflections induced by the oxygen sublattice) (b-d). One of the expected positions for strong reflections that should have been observed for the case of $\mathrm{PdO}(001)$ bulk epitaxial oxide growth is marked with the arrow in panel (b). A cartoon model of Stranski-Krastanov mechanism of epitaxial growth (panel (f)) and the reciprocal in-plane unit cell basis for three crystallographic planes of bulk PdO corresponding to the considered directions of $\mathrm{PdO}$ epitaxial growth (panel (e)). Note that all three basis are colinear and are depicted under an angle to each other for the purpose of visibility. 
also under $\mathrm{CO}$ oxidation conditions the same growth mode is found. Along with the bulk $\mathrm{PdO}$ Bragg reflections we observe the surface oxide diffraction rods, which would disappear in the case of Vollmer-Weber 3D-islands growth since there would be no surface oxide. In the case of Frank-van der Merwe multi-layer film growth the in-plane positions of superstructure diffraction rods would have changed for the thick film due to the intrinsically different inplane periodicity of bulk $\mathrm{PdO}(101)$, which has a rectangular unit cell versus the square unit cell of the $(\sqrt{5} \times \sqrt{5}) \mathrm{R} 27^{\circ}-\mathrm{PdO}(101)$ surface oxide conditioned by superstructure-substrate interaction. For a thin epitaxial film up to a certain thickness this interaction could preserve the in-plane periodicity and the change might not occur. However, in that case diffraction features would be induced by the same structure and we would then expect the epitaxial PdO bulk reflections appearing exactly on the superstructure diffraction rods rather then in their vicinity as is observed. The fact that the PdO Bragg reflections do not coincide with superstructure diffraction rods reveals a slight difference in the in-plane geometry of the unit cell for the surface and the epitaxial PdO, which is a common situation conditioned by relaxation effects. In the present case the unit cell of the surface oxide is not perfectly square but slightly distorted, which results in a small shift of superstructure diffraction rods in the hk-plane. Bulk PdO Bragg reflections appear closer to the expected positions for the square unit cell. The fact that the unit cell of the surface oxide is not square allows us to conclude that the oxide structure is affected by lateral interactions within the oxide layer, promoting the structure of bulk $\mathrm{PdO}$ rather than following the substrate periodicity. The detailed analysis of this observation will be given in a separate publication.

An epitaxial PdO growth has been reported in a previous SXRD study also under CO oxidation conditions in semi-realistic environments. ${ }^{22}$ Furthermore, an epitaxial PdO growing with the [101] crystallographic direction perpendicular to the surface of the substrate has also been reported for $\mathrm{Pd}(111)^{8,26}$ although it was observed that $\mathrm{PdO}(001)$ may coexist with $\mathrm{PdO}(101)$ by SXRD. ${ }^{11}$ The crystallographic orientation is of interest because of the presence of the oxygen under-coordinated $\mathrm{Pd}$ atoms on the $\mathrm{PdO}(101)$ surface, which have 
been assigned to be the sites responsible for catalytic activity. ${ }^{6,21,27,28}$

In an attempt to analyze the diffraction pattern further we calculated the positions of the Bragg reflections for three different epitaxial growth directions. As can be clearly seen in Fig. 3(d) the $\mathrm{PdO}(100)$ model does not fit the experimental data since the outof-plane periodicity of the reciprocal lattice in this case is completely different from the experimentally observed pattern. Thus the presence of (100)-oriented PdO islands can be disqualified. Interestingly, the growth of $\mathrm{PdO}(100)$ was unambiguously detected for (100) oriented facets of $\mathrm{Pd}$ nanoparticles grown on $\mathrm{MgAl}_{2} \mathrm{O}_{4}$ substrate. ${ }^{29}$

The calculated positions of Bragg reflections for the case of epitaxial $\mathrm{PdO}(101)$ coincide with the positions of reflections, which we observe in the recorded diffraction pattern. However, from a first glance it is not obvious whether PdO islands with only (101) or with coexisting (101) and (001) surface orientations are present. This is due to the fact that these two crystallographic orientations have the same out-of-plane periodicity for the Bragg reflections and the $\mathrm{PdO}(001)$ has a unit cell, which is half the length along one in-plane direction in real space resulting in a twice as long unit cell vector in reciprocal space as compared to $\mathrm{PdO}(101)$ (see Fig. 3(e)). This means that $\mathrm{PdO}(001)$ reflections can only explain part of the experimentally observed Bragg spots but could nevertheless be present in the diffraction pattern merging with reflections caused by $\operatorname{PdO}(101)$, which is clearly present on the surface since only this orientation explains all observed Bragg reflections. However, a thorough surveillance of the experimental data reveals the absence of some strong reflections that should be observed in the case of $\operatorname{PdO}(001)$ bulk epitaxial oxide growth, for example, the one marked with the arrow in Fig. 3(b). The fact of its absence allows us to conclude that the epitaxial PdO growth with only [101] crystallographic orientation perpendicular to the surface of the substrate takes place.

To evaluate the size of the $\mathrm{PdO}$ islands, we extracted the values of the structure factors from the surface oxide diffraction rods and the epitaxial bulk PdO Bragg reflections. One example of extracted data, for the diffraction $\operatorname{rod}(-0.8 ; 0.4 ; l)$, is shown in Fig. 4. After 

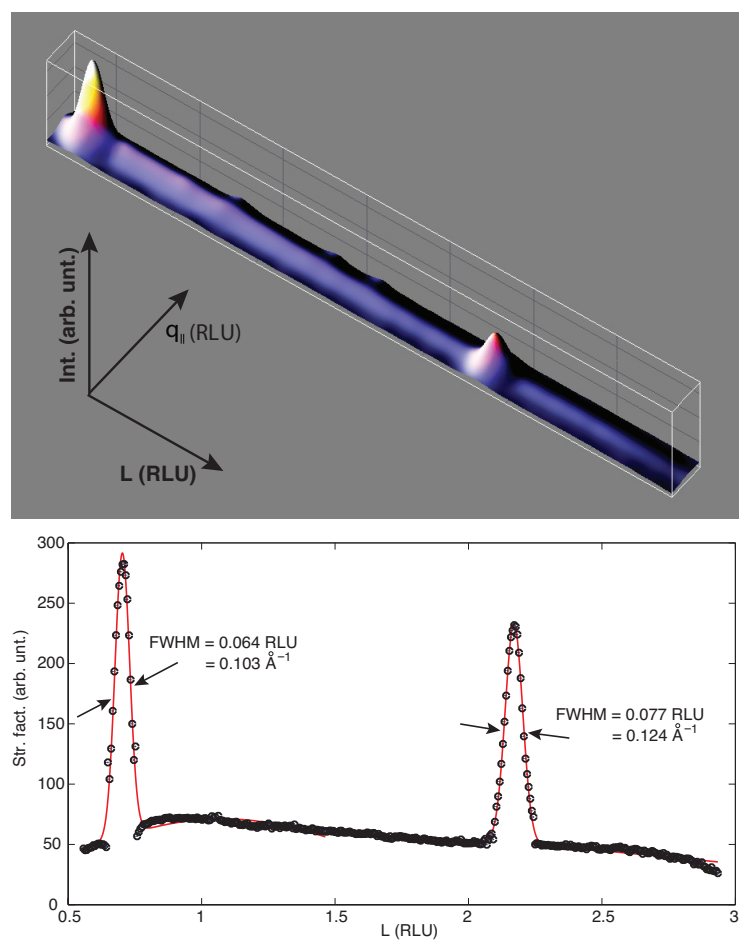

Figure 4: (Color online) 3D (top) and 2D (bottom) representation of structure factor values extracted from the experimental data for the superstructure diffraction rod $(-0.8 ; 0.4 ; l)$ fitted with Gaussian function (red line).

fitting the bulk $\mathrm{PdO}$ reflections with a Gaussian function and extracting the FWHM values for all recorded diffraction rods we estimate the average size of the epitaxial $\mathrm{PdO}$ islands to be about $52 \AA \pm 15 \AA$ in height and about $470 \AA \pm 140 \AA$ in width. This estimation is in a good agreement with results from experimental data fitting using the ANA-ROD software, ${ }^{30}$ which allows for calculating structure factor values based on a proposed structural model of a surface. The best agreement with the data is found for the $\operatorname{Pd}(100)$ surface covered with $0.8 \mathrm{ML}$ of $(\sqrt{5} \times \sqrt{5}) \mathrm{R} 27^{\circ}-\mathrm{PdO}(101)$ and additional bulk $\mathrm{PdO}(101)$ islands of 20 atomic layers $(52.8 \stackrel{\circ}{A})$ height epitaxially growing on top of the surface oxide.

When following the surface structure dynamics in the gradually changing gas environment we do not observe the appearance of Bragg reflections conditioned by the growth of epitaxial $\mathrm{PdO}$ islands. This is due to the fact that the diffraction patterns are recorded at a sample azimuthal position where such reflections do not appear. Because of restrictions in time for the present measurements, we did not perform the same experiment at the angle at which 
the Bragg reflections from the epitaxial PdO could be observed. The measurements in a constant gas flow, however, indicate that the epitaxial PdO grows under conditions similar to those existing during the dynamic measurements at a certain gas composition, although only a limited time.

Under the conditions with relatively high excess of $\mathrm{O}_{2}$ the $\mathrm{Pd}(100)$ surface is covered with a film of polycrystalline $\mathrm{PdO}$. This is demonstrated in the recorded diffraction pattern by the appearance of powder diffraction rings characteristic for a polycrystalline structure. The fact that a well ordered epitaxial oxide transforms into disordered polycrystalline phase tells us about a kinetic nature of the surface structural transformation. Possibly, the diffusion time of oxygen atoms becomes too long upon thickening of oxide phase on the surface, which leads to a non-uniform growth mode.

The positions and the relative intensity of the rings match perfectly the results of diffraction studies of a pure $\mathrm{PdO}$ powder. ${ }^{31}$ The fact that we do not observe the $\mathrm{Pd}(100)$ crystal truncation rods tells us about the roughening of the $\operatorname{Pd}(100)$ surface, which has been already demonstrated in ref. ${ }^{13}$ The detailed study of polycristalline PdO film was not the main focus of the current work, however, a coarse estimation of a monocrystallite size was performed using Sherrer's equation with the 0.9 value of the shape factor. The FWHM values for the diffraction rings are extracted from the recorded HESXRD patterns resulting in a monocrystallite mean size on the order of $200 \stackrel{\AA}{A}$.

Interestingly, when we change the gas composition over the sample from 1:2 to 1:1 ratio of $\mathrm{O}_{2}$ to $\mathrm{CO}$ and follow the surface structure dynamics, for a short period of time we see both $(\sqrt{5} \times \sqrt{5}) \mathrm{R} 27^{\circ}-\mathrm{PdO}(101)$ diffraction rods and $\mathrm{PdO}$ powder rings in the diffraction pattern. Such a pattern means that these two structures coexist on the surface for a relatively short time while the oxygen concentration over the surface gradually increases. This may indicate that the polycrystalline $\mathrm{PdO}$ before covering the entire surface starts to grow locally as has been shown previously for $\mathrm{Pd}(100)$ single crystal exposed to pure oxygen and studied ex situ. $^{23}$ 


\section{Conclusions}

Under conditions relevant to the CO oxidation reaction the surface of $\mathrm{Pd}(100)$ single crystal, acting as a model catalyst, has been shown to go through different structural states depending on the relative concentration of the reactants, which was changed from an excess of CO to an excess of $\mathrm{O}_{2}$. The notable changes are observed in three cases. In a close to stoichiometric ratio of $\mathrm{CO}$ and $\mathrm{O}_{2}$ when the mass spectrometer indicates an increase in catalytic activity, the diffraction patterns reveal the presence of $(\sqrt{5} \times \sqrt{5}) \mathrm{R} 27^{\circ}-\mathrm{PdO}(101)$ surface oxide covering the $\mathrm{Pd}(100)$ surface. Upon switching to reaction conditions with a low excess of $\mathrm{O}_{2}$ the experimental data show epitaxial growth of $\mathrm{PdO}$ islands on top of the surface oxide according to Stranski-Krastanov mechanism of growth. The island's crystallographic orientation was found to be $[101] \mathrm{PdO} \|[100] \mathrm{Pd}$ with the average size estimated to be about $52 \AA \pm 15 \AA$ in

height and about $470 \AA \pm 140 \AA$ in width. Under conditions with a high excess of $\mathrm{O}_{2}$ the surface is covered with a thick film of polycristalline $\mathrm{PdO}$ with a mean size of monocrystallites on the order of $200 \AA$. The studies of surface dynamics show that before the formation of a complete polycristalline film the disordered $\mathrm{PdO}$ coexists with the surface oxide. It is assigned to a non-uniform growth of powder-like $\mathrm{PdO}$, which starts to grow locally on the surface.

\section{Acknowledgement}

This work is done within the Röntgen-Ångström cluster "Catalysis on the atomic scale" financed by the Swedish research council (Project nr 349-2011-6491). Financial support by BMBF (Project Nr. 05K10PS1 "NanoXcat") is gratefully acknowledged.

\section{References}

(1) Ertl, G.; Knözinger, H.; Weitkamp, J. Handbook of heterogeneous catalysis; Wiley-VCH: Weinheim, 2008. 
(2) Freund, H. J.; Meijer, G.; Scheffler, M.; Schlögl, R.; Wolf, M. CO oxidation as a prototypical reaction for heterogeneous processes. Angew. Chem., Int. Ed. 2011, 50, 1006410094.

(3) Kašpar, J.; Fornasiero, P.; Hickey, N. Automotive catalytic converters: current status and some perspectives. Catal. Today 2003, 7r, 419-449.

(4) Russell, A.; Epling, W. S. Diesel oxidation catalysts. Cat. Rev. - Sci. Eng. 2011, 53, $337-423$.

(5) Goodman, D. W. Catalysis: from single crystals to the "real world". Surf. Sci. 1994, 299-300, 837-848.

(6) Weaver, J. F. Surface chemistry of late transition metal oxides. Chem. rev. 2013, 113, 4164-4215.

(7) Lundgren, E.; Gustafson, J.; Mikkelsen, A.; Andersen, J. N.; Stierle, A.; Dosch, H.; Todorova, M.; Rogal, J.; Reuter, K.; Scheffler, M. Kinetic hindrance during the initial oxidation of $\operatorname{Pd}(100)$ at ambient pressures. Phys. Rev. Lett. 2004, 92, 046101.

(8) Kan, H. H.; Weaver, J. F. A PdO(101) thin film grown on $\mathrm{Pd}(111)$ in ultrahigh vacuum. Surf. Sci. 2008, 602, L53-L57.

(9) Westerström, R.; Weststrate, C. J.; Gustafson, J.; Mikkelsen, A.; Schnadt, J.; Andersen, J. N.; Lundgren, E.; Seriani, N.; Mittendorfer, F.; Kresse, G. et al. Lack of surface oxide layers and facile bulk oxide formation on $\mathrm{Pd}(110)$. Phys. Rev. B 2009, 80, 125431.

(10) Gao, F.; Wang, Y.; Cai, Y.; Goodman, D. W. CO oxidation on Pt-group metals from ultrahigh vacuum to near atmospheric pressures. 2. Palladium and platinum. J. Phys. Chem. C 2009, 113, 174-181.

(11) Kasper, N.; Nolte, P.; Stierle, A. Stability of surface and bulk oxides on Pd(111) revisited by in situ X-ray diffraction. J. Phys. Chem. C 2012, 116, 21459-21464. 
(12) Todorova, M.; Lundgren, E.; Blum, V.; Mikkelsen, A.; Gray, S.; Gustafson, J.; Borg, M.; Rogal, J.; Reuter, K.; Andersen, J. N. et al. The $\operatorname{Pd}(100)-(\sqrt{5} \times \sqrt{5}) \mathrm{R}_{2} 7^{\circ}-\mathrm{O}$ surface oxide revisited. Surf. Sci. 2003, 541, 101-112.

(13) Stierle, A.; Kasper, N.; Dosch, H.; Lundgren, E.; Gustafson, J.; Mikkelsen, A.; Andersen, J. N. A surface X-ray study of the structure and morphology of the oxidized Pd(001) surface. J. Chem. Phys. 2005, 122, 044706.

(14) Kostelník, P.; Seriani, N.; Kresse, G.; Mikkelsen, A.; Lundgren, E.; Blum, V.; Šikola, T.; Varga, P.; Schmid, M. The surface oxide: a LEED, DFT and STM study. Surf. Sci. 2007, 601, 1574-1581.

(15) Seriani, N.; Harl, J.; Mittendorfer, F.; Kresse, G. A first-principles study of bulk oxide formation on $\operatorname{Pd}(100)$. J. Chem. Phys. 2009, 131, 054701.

(16) Van Rijn, R.; Balmes, O.; Felici, R.; Gustafson, J.; Wermeille, D.; Westerstrm, R.; Lundgren, E.; Frenken, J. W. M. Comment on "CO oxidation on Pt-group metals from ultrahigh vacuum to near atmospheric pressures. 2. Palladium and platinum". J. Phys. Chem. C 2010, 114, 6875-6876.

(17) Blomberg, S.; Hoffmann, M.; Gustafson, J.; Martin, N.; Fernandes, V.; Borg, A.; Liu, Z.; Chang, R.; Matera, S.; Reuter, K. et al. In situ X-ray photoelectron spectroscopy of model catalysts: at the edge of the gap. Phys. Rev. Lett. 2013, 110, 117601.

(18) Blomberg, S.; Brackmann, C.; Gustafson, J.; Aldén, M.; Lundgren, E.; Zetterberg, J. Real-time gas-phase imaging over $\mathrm{Pd}(110)$ catalyst during $\mathrm{CO}$ oxidation by means of planar laser-induced fluorescence. ACS Catal. 2015, 5, 2028-2034.

(19) Gustafson, J.; Shipilin, M.; Zhang, C.; Stierle, A.; Hejral, U.; Ruett, U.; Gutowski, O.; Carlsson, P.-A.; Skoglundh, M.; Lundgren, E. High-energy surface X-ray diffraction for fast surface structure determination. Science 2014, 343, 758-761. 
(20) Shipilin, M.; Hejral, U.; Lundgren, E.; Merte, L. R.; Zhang, C.; Stierle, A.; Ruett, U.; Gutowski, O.; Skoglundh, M.; Carlsson, P.-a. et al. Quantitative surface structure determination using in situ high-energy SXRD: surface oxide formation on $\mathrm{Pd}(100)$ during catalytic CO oxidation. Surf. Sci. 2014, 630, 229-235.

(21) Westerström, R.; Messing, M. E.; Blomberg, S.; Hellman, A.; Grönbeck, H.; Gustafson, J.; Martin, N. M.; Balmes, O.; Van Rijn, R.; Andersen, J. N. et al. Oxidation and reduction of $\operatorname{Pd}(100)$ and aerosol-deposited $\mathrm{Pd}$ nanoparticles. Phys. Rev. $B 2011,83,115440$.

(22) Van Rijn, R.; Balmes, O.; Resta, A.; Wermeille, D.; Westerström, R.; Gustafson, J.; Felici, R.; Lundgren, E.; Frenken, J. W. M. Surface structure and reactivity of $\operatorname{Pd}(100)$ during CO oxidation near ambient pressures. Phys. Chem. Chem. Phys. 2011, 13, 13167-13171.

(23) Han, J.; Zemlyanov, D. Y.; Ribeiro, F. H. Interaction of $\mathrm{O}_{2}$ with Pd single crystals in the range 1-150 Torr: surface morphology transformations. Surf. Sci. 2006, 600, $2730-2744$.

(24) van Rijn, R.; Ackermann, M.; Balmes, O.; Dufrane, T.; Geluk, A.; Gonzalez, H.; Isern, H.; de Kuyper, E.; Petit, L.; Sole, V. A. et al. Ultrahigh vacuum/high-pressure flow reactor for surface X-ray diffraction and grazing incidence small angle X-ray scattering studies close to conditions for industrial catalysis. Rev. Sci. Instrum. 2010, 81, 014101.

(25) Reichert, H.; Honkimäki, V.; Snigirev, A.; Engemann, S.; Dosch, H. A new X-ray transmission-reflection scheme for the study of deeply buried interfaces using highenergy microbeams. Physica B 2003, 336, 46 - 55.

(26) Hinojosa, J. A.; Weaver, J. F. Surface structural evolution during the thermal decomposition of a $\operatorname{PdO}(101)$ thin film. Surf. Sci. 2011, 605, 1797-1806. 
(27) Hellman, A.; Resta, A.; Martin, N. M.; Gustafson, J.; Trinchero, A.; Carlsson, P.-A.; Balmes, O.; Felici, R.; Van Rijn, R.; Frenken, J. W. M. et al. The active phase of palladium during methane oxidation. J. Phys. Chem. Lett. 2012, 3, 678-682.

(28) Martin, N. M.; Van Den Bossche, M.; Grönbeck, H.; Hakanoglu, C.; Gustafson, J.; Blomberg, S.; Arman, M. A.; Antony, A.; Rai, R.; Asthagiri, A. et al. Dissociative adsorption of hydrogen on $\mathrm{PdO}(101)$ studied by HRCLS and DFT. J. Phys. Chem. C 2013, 117, 13510-13519.

(29) Müller, P.; Hejral, U.; Rütt, U.; Stierle, A. In situ oxidation study of Pd-Rh nanoparticles on $\mathrm{MgAl}_{2} \mathrm{O}_{4}(001)$. Phys. Chem. Chem. Phys. 2014, 16, 13866-13874.

(30) Vlieg, E. ROD, a program for surface X-ray crystallography. J. Appl. Cryst. 2000, 33, 401-405.

(31) Waser, J.; Levy, H. A.; Peterson, S. W. The structure of PdO. Acta Crystallogr. 1953, $6,661-663$. 
Graphical TOC Entry

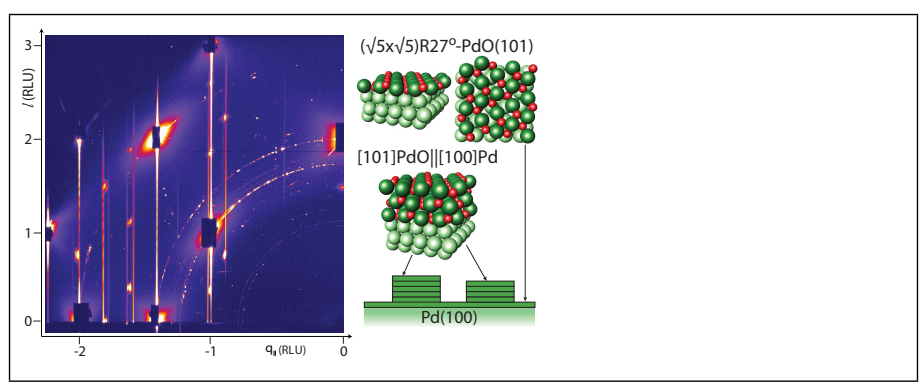

